(i)

\title{
Editorial
}

\section{Medical Discoveries: From benchtop to bedside}

Long ago, when for the first time Edward Jenner, a country doctor living in Berkeley (Gloucestershire), England, who performed the world's first vaccination in 1796, it leads to speculations that all the disease can be prevented or eradicated. With unifying efforts of Biomedical scientists along with public health workers, it was only in 1980 the small pox was ultimately eradicated from the World. Thus it took nearly 2 centuries to see the fruits of the untiring work of our researchers and healthcare manpower.

Tuberculosis, a disease considered to be incurable before 1946, found a ray of hope with discovery of Streptomycin — an antibiotic purified from Streptomyces griseus - as the first antibiotic with proven activity against Mycobacterium tuberculosis. However, biomedical scientists found that uncertainties remained about its ability to consistently cure patients, and this was closely followed by the realization that drug resistance develops rapidly when a single agent is used for the treatment of TB. The untiring efforts of scientists once again lead to development of another drug Rifampicin in 1960s which changed the scenario of treatment of TB in sanatorium to domiciliary mode. It also paved way for the scientists to realize that only randomized controlled trials are the gold standard for judging the efficacy of a new drug.

The science fictions give us an inkling that it is not impossible to think about innovative device drug or idea to change the face of healthcare. However, life of scientists working on these innovations provide us teaching tips that scientific discoveries are not abrupt but took pretty long time for fruitful translation into the clinical practice.

When a four-year old girl became the first gene therapy patient on September 14, 1990 at the NIH Clinical Center as she had adenosine deaminase (ADA) deficiency, a genetic disease which leaves her defenseless against infections, it was speculated that all genetic disorders may be cured with help of Gene therapy. Twenty five years later we are still not able to develop an effective and safe gene therapy for as common a disorder as Thalassemia.

There are ideas, there are researcher devoting their lifetime on these ideas, there are scientists who have proved others wrong and there are others who created examples of ethical medical practice using and there is generation of medical scientists using evidence based medicine in daily practice and who are looking forward to new 
discoveries and inventions. But one thing to be realized is that there is no short cut for finding cure to human diseases.

In this issue, we can visualize the long journey for the infectious diseases namely Neurocysticercosis and challenges in development of vaccine for Japanese encephalitis.

It is also heartening to note that innovations are not only needed in health care devices but also in way we prepare our future generation of physicians. Innovations are also required for detecting diseases at the nascent stage. Then there are benign breast diseases where life time efforts leads to elucidate the natural history and development of cost effective therapy. This issue is dedicated to the efforts of all biomedical scientists whose untiring work and efforts culminated in better bedside therapies and whose teaching will prove lighthouse for future generation of Indian biomedical scientists.

Dr Sanjeev Misra 